\title{
Plasma Magnesium and the Risk of Ischemic Stroke among Women
}

\author{
Sally N. Akarolo-Anthony, MD, MSc ${ }^{1,2}$, Monik C. Jiménez, ScD², Stephanie E. Chiuve, \\ $\mathrm{ScD}^{1,2}$, Donna Spiegelman, ScD ${ }^{3,4}$, Walter C. Willett, MD, $\operatorname{DrPH}^{1,3}$, and Kathryn M. Rexrode, \\ MD, MPH ${ }^{2}$ \\ ${ }^{1}$ Department of Nutrition, Harvard School of Public Health \\ 2Division of Preventive Medicine, Department of Medicine, Brigham and Women's Hospital \\ ${ }^{3}$ Department of Epidemiology, Harvard School of Public Health \\ ${ }^{4}$ Department of Biostatistics, Harvard School of Public Health
}

\begin{abstract}
Background and Purpose-Lower plasma magnesium levels may be associated with higher blood pressure and endothelial dysfunction, but sparse prospective data are available for stroke.

Methods-Among 32,826 participants in the Nurses' Health Study who provided blood samples in 1989-1990, incident ischemic strokes were identified and confirmed by medical records through 2006. We conducted a nested case-control analysis of 459 cases, matched 1:1 to controls on age, race/ethnicity, smoking status, date of blood draw, fasting status, menopausal status and hormone use. We used conditional logistic regression models to estimate the multivariable adjusted association of plasma magnesium and the risk of ischemic stroke and ischemic stroke subtypes.
\end{abstract}

Results-Median magnesium levels did not differ between ischemic stroke cases and controls (median $=0.86 \mathrm{mmol} / \mathrm{l}$ for both; $\mathrm{p}$-value $=0.14$ ). Conditional on matching factors, women in the lowest magnesium quintile had a relative risk (RR) of 1.34 (95\% confidence interval [CI]: 0.862.10, $\mathrm{p}$ trend $=0.13$ ) for total ischemic stroke, compared to women in the highest quintile. Additional adjustment for risk factors and confounders did not substantially alter the risk estimates for total ischemic stroke. Women with magnesium levels $<0.82 \mathrm{mmol} / \mathrm{l}$, had significantly greater risk of total ischemic stroke (multivariable $\mathrm{RR}=1.57$; 95\% CI: 1.09-2.27, $\mathrm{p}=0.01$ ), and thrombotic stroke (multivariable $\mathrm{RR}=1.66$; 95\% CI: $1.03-2.65, \mathrm{p}=0.03$ ) compared to women with magnesium levels $\searrow 0.82 \mathrm{mmol} / \mathrm{l}$. No significant effect modification was observed by age, body mass index, hypertension or diabetes.

Conclusions-Lower plasma magnesium levels may contribute to higher risk of ischemic stroke among women.

Corresponding author: Sally N. Akarolo-Anthony, Department of Nutrition, Harvard School of Public Health, 677 Huntington Avenue, Boston, MA 02115, USA. Tel: +1 4439857112 Fax: +1 4107061944 sna094@ mail.harvard.edu.

Disclosures: None 


\section{Keywords}

Plasma Magnesium; Ischemic Stroke

\section{Introduction}

Plasma levels of magnesium have been inversely associated with risk factors for stroke, such as hypertension ${ }^{1,2}$ and diabetes ${ }^{3,4}$. Plasma magnesium has also been inversely associated with the risk of cardiovascular outcomes including coronary heart disease ${ }^{5,6}$ and atrial fibrillation ${ }^{7}$. Lower magnesium levels may lead to stroke by initiating an inflammatory cascade which triggers oxidative responses in endothelial cells leading to vasoconstriction and thrombus formation ${ }^{8}$, or through effects on hypertension, diabetes and atrial fibrillation.

The relationship between serum magnesium and the risk of ischemic stroke has been examined in only one prospective cohort study ${ }^{9}$. Low serum magnesium levels were associated with a higher risk of ischemic stroke among men and women in the Atherosclerosis Risk in Communities (ARIC) Study, men and women in the highest plasma magnesium quartile had a 25\% (95\% CI, 5-41\%) reduced risk of ischemic stroke in models adjusted for age, sex and race; however, adjustment for hypertension and diabetes, which may possibly be biological mediators of the effects of magnesium, eliminated an association ${ }^{9}$. To provide further evidence on this relationship, we prospectively examined the association between plasma magnesium and the risk of ischemic stroke and ischemic stroke subtypes (thrombotic and embolic strokes) among women in the Nurses' Health Study (NHS).

\section{Methods}

\section{Study population}

The NHS is a prospective cohort study which began in 1976, when 121,700 female registered nurses aged 30 - 55 years at enrollment, completed questionnaires about their lifestyle factors and medical history. Follow-up questionnaires are sent to these women biennially to update this information. Between 1989 and 1990, 32,826 women in this study cohort provided blood samples; of these, 18,743 provided a second blood sample between 2000 and 2001. The women who provided a blood sample did not differ appreciably from those who did not. ${ }^{10}$ Blood samples archived in continuously monitored liquid nitrogen freezers at -80 degrees Celsius.

We carried out a nested case-control study of ischemic stroke among the 32,826 women who provided a blood sample. Cases and controls were both required to be free of cancer or prior cardiovascular disease at the time of blood collection. Incident ischemic stroke cases were matched 1:1 to controls who remained free of stroke prior to the case date, with matching by age ( \pm 2 years), race/ethnicity (white/African-American/Asian/Hispanic/other/unknown), smoking (never, past and current), date of blood draw, fasting status, menopausal status and hormone use (yes/no). This study was approved by the institutional review board at Brigham and Women's Hospital and informed consent was obtained from all participants. 


\section{Ascertainment of Plasma Magnesium}

Matched ischemic stroke case-control pairs were shipped to the laboratory in the same batch. Magnesium was measured by colorimetric assay on an Hitachi 917 analyzer (Roche Diagnostics, Indianapolis, IN). The coefficient of variation for plasma magnesium was $4 \%$ and the intraclass correlation between two blood samples collected from the same women was 0.63 for samples obtained 2 to 3 years apart and 0.39 for samples obtained 10 years apart. Total cholesterol (CV of 4\%), LDL and HDL cholesterol (CV of 4\%), C-reactive protein ( $\mathrm{CV}$ of $2 \%$ ), and glycosylated hemoglobin ( $\mathrm{CV}$ of $4 \%$ ), were measured in the Clinical and Epidemiological Research Laboratory at Children's Hospital (Boston, MA) for all cases and controls.

\section{Ascertainment of Stroke}

Women who reported a nonfatal stroke on a follow-up questionnaire were asked for permission to review their medical records. Medical records were reviewed by physicians blinded to the exposure status. Fatal strokes were initially ascertained by reports from relatives or postal authorities and a search of the National Death Index and were then documented by medical records and death certificates.

Strokes were confirmed according to the criteria of the National Survey of Stroke which requires a constellation of neurological deficits of sudden or rapid onset lasting $\geq 24$ hours or until death. Strokes were regarded as incident if they occurred after the date of return of the 1980 questionnaire but before June 2006. Strokes were classified as ischemic stroke due to thrombotic or embolic occlusion of a cerebral artery with imaging data from computed tomography (CT) or magnetic resonance imaging (MRI); $97 \%$ of the cases had a CT or MRI. Thrombotic strokes were defined as infarction involving the cortical artery regions in the cerebrum and the cerebellum (cortex and subcortical areas) or the focal, small and deep areas such as the internal capsule, corona radiate, basal ganglia and brainstem, without involvement of cortex. Strokes were defined as embolic if evidence of an embolic source was present in the medical record and if imaging studies and/or neurology consult supported the diagnosis ${ }^{11}$. In the setting of incomplete evidence or competing etiologies where type could not be assigned, the strokes were considered unclassified ischemic stroke.

\section{Statistical Analyses}

A total of 459 confirmed ischemic stroke cases (303 thrombotic, 129 embolic and 27 unclassified ischemic strokes) and 459 controls had magnesium levels available for analyses. Quintiles of plasma magnesium were created based on the distribution of plasma magnesium among the controls, and assigned cases to each quintile based on their plasma magnesium levels. Magnesium levels were also dichotomized $(<0.82 \mathrm{mmol} / \mathrm{l}$ versus $\searrow 0.82$ $\mathrm{mmol} / \mathrm{l}$ ) to approximate clinically low levels, and compare the lowest quintile to all other quintiles. The means and proportions of baseline characteristics, cardiovascular risk factors, and biomarkers across quintiles of plasma magnesium were calculated among the cases and controls; we conducted tests of significance to compare the means and proportions between cases and controls, using Mantel-Haenszel and Fisher's exact tests. 
Multivariable conditional logistic regression was used to examine the association between plasma magnesium and the risk of ischemic stroke and ischemic stroke subtypes (thrombotic and embolic strokes). Three multivariable models were created, model 1 was conditional on matching factors only: age, race/ethnicity, smoking, date of blood draw, fasting status, menopausal status and hormone use; model 2 was adjusted for lifestyle risk factors: alcohol, body mass index (BMI), physical activity, aspirin and thiazide diuretic use; model 3 was further adjusted for potential mediators: HbA1c, history of diabetes, hypertension, coronary heart disease and total/high density lipoprotein cholesterol (total/HDL cholesterol). For each model, we derived relative risks (RR's) and 95\% confidence intervals (CI's). Effect modification by age, BMI, hypertension and diabetes was tested and significance of interactions was assessed using the likelihood ratio tests. Sensitivity analyses were conducted to calculate adjusted RRs and 95\% CIs for measurement error correction in plasma magnesium, using samples collected approximately 10 years apart. We also examined the possibly non-linear relation between plasma magnesium and ischemic stroke with likelihood ratio tests, comparing the model with only the linear term to the model with the linear and cubic spline terms. All analyses were conducted with SAS for UNIX statistical software (version 9.2; SAS Institute).

\section{Results}

Among the 459 women who developed an ischemic stroke, the mean age at baseline in 1990 was 60.8 years, while the mean age at stroke diagnosis was 71 years. The differences between cases and controls in cardiovascular disease (CVD) risk factors for ischemic stroke are shown in Table 1. Women in the lowest quintile of plasma magnesium were more likely to consume more alcohol, be current smokers, use thiazide diuretics, postmenopausal hormone therapy, have hypertension and diabetes, compared to women in the highest quintile (Table 2). The median magnesium levels in the lowest and highest quintiles, based on the distribution in the controls, were $0.78 \mathrm{mmol} / 1$ and $0.95 \mathrm{mmol} / 1$ respectively. The median magnesium levels did not differ between the ischemic stroke cases and controls $(0.86 \mathrm{mmol} / \mathrm{l}$ in each, p-value 0.14$)$ in univariate analyses.

In models conditional on matching factors (Table 3), there was no significant association between plasma magnesium and the risk of total ischemic stroke. The RR and 95\% CI for the lowest quintile compared to the highest quintile was 1.34 (0.86-2.10), p-trend 0.13. This risk was similar after adjusting for other lifestyle risk factors (RR 1.24 [0.78 - 1.98], ptrend 0.29) including alcohol intake, BMI, physical activity, aspirin and thiazide diuretics use. When further adjusted for $\mathrm{HbAlc}$, history of diabetes, history of hypertension, coronary heart disease, total/HDL cholesterol, the risk estimates remained similar (RR 1.34 [0.82 2.17], p-trend 0.19). Next, we examined the association of plasma magnesium with thrombotic and embolic strokes, the RR and 95\% CI for the lowest vs highest quintile was $1.63(0.89-2.98)$, p-trend 0.09 for thrombotic stroke and $1.12(0.39-3.19)$, p-trend 0.72 for embolic stroke, in the fully adjusted models.

When magnesium levels were dichotomized comparing the lowest quintile to all other categories, the RR and $95 \%$ CI for magnesium levels $<0.82 \mathrm{mmol} / \mathrm{l}$ compared to those $\searrow 0.82$ $\mathrm{mmol} / \mathrm{l}$ was $1.64(1.17-2.31)$, conditional on matching factors only (Table 4$)$. When we 
adjusted for confounding by other lifestyle risk factors, this risk estimate was not substantially altered. In the fully adjusted model (model 3), the RR and 95\% CI for total stroke was $1.57(1.09-2.27)$. The estimates were similar for thrombotic stroke, 1.66 (1.03 2.65).

Additional analyses correcting for measurement error did not yield substantially different results. Potential effect modification of the association between plasma magnesium and risk of ischemic stroke by age, BMI, hypertension and diabetes was examined in stratified analysis. No significant effect modification was observed by any of these variables ( $p$ > 0.05 ) but power may be limited.

\section{Discussion}

In this prospective study, plasma magnesium levels were not associated with the risk of ischemic stroke in women across the full distribution of plasma magnesium. However, women with magnesium levels $<0.82 \mathrm{mmol} / \mathrm{l}$ had a $57 \%$ (95\% CI: $9 \%$ - 127\%) higher risk of ischemic stroke and this association remained unchanged after controlling for other factors associated with magnesium levels and stroke risk.

Several cross-sectional and retrospective case-control studies have observed that serum magnesium levels are lower in individuals with acute stroke compared to healthy controls $^{12-14}$. However, in these studies, magnesium was not measured prior to the stroke diagnosis and thus hypomagnesaemia may have been a consequence rather than a cause of stroke in these patients. Nonetheless, magnesium may influence stroke severity and outcome; individuals with lower magnesium levels had worse post-stroke prognosis ${ }^{13}, 15$. Early data from a small randomized clinical trial suggested a benefit of intravenous magnesium on acute stroke prognosis and outcome ${ }^{16}$, but results from the Intravenous Magnesium Efficacy in Stroke (IMAGES) trial ${ }^{17}$ as well as preliminary results from the FAST-MAG trial presented at the 2014 American Stroke Association's International Stroke Conference failed to show any clinical benefit of intravenous magnesium infusion on stroke outcomes in the acute stroke setting.

To the best of our knowledge, only one other prospective study has examined magnesium levels and risk of ischemic stroke. In the Atherosclerosis Risk in Communities (ARIC) study based on 577 ischemic stroke cases in men and women with 16 years of follow up, serum magnesium levels were inversely associated with ischemic stroke incidence. However, adjustment for hypertension and diabetes attenuated risk ratios to non-significant levels (RR comparing high to low serum magnesium quartiles, 1.04; 95\% CI, $0.82-1.32, \mathrm{p}=0.99$ ), suggesting that these factors may have mediated the association. In our study, we found increased risk of ischemic stroke among women with lower magnesium levels, even after controlling for hypertension and diabetes. The difference between our findings and those of ARIC may be explained by differences in the populations. The ARIC study included white and black men and women who had a mean age of 54 years at baseline, while our study population consisted of only female nurses who were predominantly white with a mean age of approximately 60 years at baseline. 
Further, our data are generally in accordance with the findings on dietary magnesium, although the correlation between dietary and plasma levels is poor $(r=0.02)^{5}$. In a metaanalysis of 7 prospective studies, dietary magnesium intake was inversely associated with the risk of stroke, an increase in intake of $100 \mathrm{mg} /$ day was associated with a 9\% significant reduction in risk of ischemic stroke ${ }^{18}$. Magnesium-rich foods such as green leafy vegetables ${ }^{19,20}$, whole grains ${ }^{21,22}$ and coffee ${ }^{23}$ have also been associated with reduced risk of ischemic stroke in the Nurses' Health Study and other cohorts.

The best method of ascertaining magnesium status remains unclear. Plasma magnesium accounts for only $1 \%$ of whole body magnesium concentration ${ }^{24}$ but has been shown to be fairly strongly correlated with intracellular free magnesium levels $(r=0.54)^{25}$. As magnesium is under tight homeostatic control, dietary magnesium may be a poor estimate of biologically active magnesium; thus, plasma magnesium may be a better exposure to estimate the true association between magnesium and stroke.

Several potential mechanisms may mediate a reduced risk of ischemic stroke by magnesium. Hypertension and diabetes, known risk factors for stroke, were inversely associated with plasma levels of magnesium ${ }^{2}$. In experimental studies, increased plasma magnesium levels appeared to reduce blood pressure by blocking calcium channels, thus attenuating agonist induced vasoconstriction, decreasing vascular resistance and increasing the capacitance function of cerebral arteries ${ }^{26-29}$. High magnesium levels have been shown to have beneficial effects on insulin resistance, glucose metabolism and risk of type 2 diabetes ${ }^{30}$. A small randomized clinical trial showed that magnesium supplementation $(500 \mathrm{mg}$ magnesium citrate daily for 4 weeks) was associated with reduced insulin concentrations ${ }^{31}$.

In addition, magnesium has been shown to inhibit arterial thrombus formation in animal studies $^{32,33}$ thus low plasma magnesium levels may be associated with the risk of thrombus formation in humans. In previous studies, serum magnesium levels were inversely associated with von Willebrand factor levels ${ }^{9}$, and von Willebrand factor levels were positively associated with the incidence of ischemic stroke ${ }^{34}$. Although low levels of serum magnesium have been associated with higher risk of atrial fibrillation ${ }^{7,35}$ which is a potent risk factor for embolic stroke ${ }^{36}$, our study was underpowered to evaluate the hypothesis of an anti-arrhythmic effect of magnesium as a potential pathway for reducing the risk of ischemic stroke.

This study has several strengths including its nested case control design, prospectively collected blood samples, and careful stroke outcome assessment based on medical records. However, the study is limited by using a single assessment of plasma magnesium at baseline to examine the relationship between plasma magnesium and ischemic stroke and there may be substantial variation in levels over time; only a modest intraclass correlation between 2 plasma sample measurements approximately 10 years apart was observed $(r=0.39)$. Furthermore, we studied a population of women who were predominantly Caucasian; therefore our results may not be generalizable to men or other racial and ethnic groups. 
In conclusion, the results of this study suggest that low plasma magnesium may be associated with increased risk of ischemic stroke. If confirmed, our findings may have significant public health impact as magnesium deficiency is potentially modifiable.

\section{Acknowledgments}

Sources of Funding: This project was funded by NIH grants: HL088521, HL34594, CA87969, and CA 49449.

\section{References}

1. Peacock JM, Folsom AR, Arnett DK, Eckfeldt JH, Szklo M. Relationship of serum and dietary magnesium to incident hypertension: The atherosclerosis risk in communities (aric) study. Ann Epidemiol. 1999; 9:159-165. [PubMed: 10192647]

2. Ma J, Folsom AR, Melnick SL, Eckfeldt JH, Sharrett AR, Nabulsi AA, et al. Associations of serum and dietary magnesium with cardiovascular disease, hypertension, diabetes, insulin, and carotid arterial wall thickness: The aric study. Atherosclerosis risk in communities study. J Clin Epidemiol. 1995; 48:927-940. [PubMed: 7782801]

3. Simmons D, Joshi S, Shaw J. Hypomagnesaemia is associated with diabetes: Not pre-diabetes, obesity or the metabolic syndrome. Diabetes Res Clin Pract. 2010; 87:261-266. [PubMed: 20004036]

4. Xu J, Xu W, Yao H, Sun W, Zhou Q, Cai L. Associations of serum and urinary magnesium with the pre-diabetes, diabetes and diabetic complications in the chinese northeast population. PLoS One. 2013; 8:e56750. [PubMed: 23418599]

5. Chiuve SE, Sun Q, Curhan GC, Taylor EN, Spiegelman D, Willett WC, et al. Dietary and plasma magnesium and risk of coronary heart disease among women. J Am Heart Assoc. 2013; 2:e000114. [PubMed: 23537810]

6. Joosten MM, Gansevoort RT, Mukamal KJ, van der Harst P, Geleijnse JM, Feskens EJ, et al. Urinary and plasma magnesium and risk of ischemic heart disease. Am J Clin Nutr. 2013; 97:1299_ 1306. [PubMed: 23485414]

7. Khan AM, Lubitz SA, Sullivan LM, Sun JX, Levy D, Vasan RS, et al. Low serum magnesium and the development of atrial fibrillation in the community: The framingham heart study. Circulation. 2013; 127:33-38. [PubMed: 23172839]

8. Wolf FI, Trapani V, Simonacci M, Ferre S, Maier JA. Magnesium deficiency and endothelial dysfunction: Is oxidative stress involved? Magnes Res. 2008; 21:58-64. [PubMed: 18557135]

9. Ohira T, Peacock JM, Iso H, Chambless LE, Rosamond WD, Folsom AR. Serum and dietary magnesium and risk of ischemic stroke: The atherosclerosis risk in communities study. Am J Epidemiol. 2009; 169:1437-1444. [PubMed: 19372211]

10. Larsson SC, Virtamo J, Wolk A. Potassium, calcium, and magnesium intakes and risk of stroke in women. Am J Epidemiol. 2011; 174:35-43. [PubMed: 21540318]

11. Iso $\mathrm{H}$, Rexrode $\mathrm{K}$, Hennekens $\mathrm{CH}$, Manson JE. Application of computer tomography-oriented criteria for stroke subtype classification in a prospective study. Ann Epidemiol. 2000; 10:81-87. [PubMed: 10691061]

12. Altura BT, Memon ZI, Zhang A, Cheng TP, Silverman R, Cracco RQ, et al. Low levels of serum ionized magnesium are found in patients early after stroke which result in rapid elevation in cytosolic free calcium and spasm in cerebral vascular muscle cells. Neurosci Lett. 1997; 230:3740. [PubMed: 9259458]

13. Feng P, Niu X, Hu J, Zhou M, Liang H, Zhang Y, et al. Relationship of serum magnesium concentration to risk of short-term outcome of acute ischemic stroke. Blood Press. 2013; 22:297301. [PubMed: 23339516]

14. Cojocaru IM, Cojocaru M, Burcin C, Atanasiu NA. Serum magnesium in patients with acute ischemic stroke. Rom J Intern Med. 2007; 45:269-273. [PubMed: 18333360] 
15. Bayir A, Ak A, Kara H, Sahin TK. Serum and cerebrospinal fluid magnesium levels, glasgow coma scores, and in-hospital mortality in patients with acute stroke. Biol Trace Elem Res. 2009; 130:7-12. [PubMed: 19165425]

16. Lampl Y, Gilad R, Geva D, Eshel Y, Sadeh M. Intravenous administration of magnesium sulfate in acute stroke: A randomized double-blind study. Clin Neuropharmacol. 2001; 24:11-15. [PubMed: 11290876]

17. Muir KW, Lees KR, Ford I, Davis S. Magnesium for acute stroke (intravenous magnesium efficacy in stroke trial): Randomised controlled trial. Lancet. 2004; 363:439-445. [PubMed: 14962524]

18. Larsson SC, Orsini N, Wolk A. Dietary magnesium intake and risk of stroke: A meta-analysis of prospective studies. Am J Clin Nutr. 2012; 95:362-366. [PubMed: 22205313]

19. Joshipura KJ, Ascherio A, Manson JE, Stampfer MJ, Rimm EB, Speizer FE, et al. Fruit and vegetable intake in relation to risk of ischemic stroke. JAMA. 1999; 282:1233-1239. [PubMed: 10517425]

20. Larsson SC, Virtamo J, Wolk A. Total and specific fruit and vegetable consumption and risk of stroke: A prospective study. Atherosclerosis. 2013; 227:147-152. [PubMed: 23294925]

21. Liu S, Manson JE, Stampfer MJ, Rexrode KM, Hu FB, Rimm EB, et al. Whole grain consumption and risk of ischemic stroke in women: A prospective study. JAMA. 2000; 284:1534-1540. [PubMed: 11000647]

22. Steffen LM, Jacobs DR Jr, Stevens J, Shahar E, Carithers T, Folsom AR. Associations of wholegrain, refined-grain, and fruit and vegetable consumption with risks of all-cause mortality and incident coronary artery disease and ischemic stroke: The atherosclerosis risk in communities (aric) study. Am J Clin Nutr. 2003; 78:383-390. [PubMed: 12936919]

23. Lopez-Garcia E, Rodriguez-Artalejo F, Rexrode KM, Logroscino G, Hu FB, van Dam RM. Coffee consumption and risk of stroke in women. Circulation. 2009; 119:1116-1123. [PubMed: 19221216]

24. Wary C, Brillault-Salvat C, Bloch G, Leroy-Willig A, Roumenov D, Grognet JM, et al. Effect of chronic magnesium supplementation on magnesium distribution in healthy volunteers evaluated by 31p-nmrs and ion selective electrodes. Br J Clin Pharmacol. 1999; 48:655-662. [PubMed: 10594466]

25. Ryzen E, Servis KL, DeRusso P, Kershaw A, Stephen T, Rude RK. Determination of intracellular free magnesium by nuclear magnetic resonance in human magnesium deficiency. $\mathrm{J}$ Am Coll Nutr. 1989; 8:580-587. [PubMed: 2621295]

26. Griendling KK, Rittenhouse SE, Brock TA, Ekstein LS, Gimbrone MA Jr, Alexander RW. Sustained diacylglycerol formation from inositol phospholipids in angiotensin ii-stimulated vascular smooth muscle cells. J Biol Chem. 1986; 261:5901-5906. [PubMed: 3084474]

27. Touyz RM, Yao G. Modulation of vascular smooth muscle cell growth by magnesium-role of mitogen-activated protein kinases. J Cell Physiol. 2003; 197:326-335. [PubMed: 14566962]

28. Yoshimura M, Oshima T, Matsuura H, Ishida T, Kambe M, Kajiyama G. Extracellular mg2+ inhibits capacitative ca2+ entry in vascular smooth muscle cells. Circulation. 1997; 95:2567-2572. [PubMed: 9184588]

29. Resnick LM, Gupta RK, DiFabio B, Barbagallo M, Mann S, Marion R, et al. Intracellular ionic consequences of dietary salt loading in essential hypertension. Relation to blood pressure and effects of calcium channel blockade. J Clin Invest. 1994; 94:1269-1276. [PubMed: 8083368]

30. Paolisso G, Barbagallo M. Hypertension, diabetes mellitus, and insulin resistance: The role of intracellular magnesium. Am J Hypertens. 1997; 10:346-355. [PubMed: 9056694]

31. Chacko SA, Sul J, Song Y, Li X, LeBlanc J, You Y, et al. Magnesium supplementation, metabolic and inflammatory markers, and global genomic and proteomic profiling: A randomized, doubleblind, controlled, crossover trial in overweight individuals. Am J Clin Nutr. 2011; 93:463-473. [PubMed: 21159786]

32. Gertz SD, Wajnberg RS, Kurgan A, Uretzky G. Effect of magnesium sulfate on thrombus formation following partial arterial constriction: Implications for coronary vasospasm. Magnesium. 1987; 6:225-235. [PubMed: 3441141] 
33. Ravn HB, Kristensen SD, Hjortdal VE, Thygesen K, Husted SE. Early administration of intravenous magnesium inhibits arterial thrombus formation. Arterioscler Thromb Vasc Biol. 1997; 17:3620-3625. [PubMed: 9437213]

34. Folsom AR, Rosamond WD, Shahar E, Cooper LS, Aleksic N, Nieto FJ, et al. Prospective study of markers of hemostatic function with risk of ischemic stroke. The atherosclerosis risk in communities (aric) study investigators. Circulation. 1999; 100:736-742. [PubMed: 10449696]

35. Misialek JR, Lopez FL, Lutsey PL, Huxley RR, Peacock JM, Chen LY, et al. Serum and dietary magnesium and incidence of atrial fibrillation in whites and in african americans--atherosclerosis risk in communities (aric) study. Circ J. 2013; 77:323-329. [PubMed: 23047297]

36. Wolf PA, Abbott RD, Kannel WB. Atrial fibrillation as an independent risk factor for stroke: The framingham study. Stroke. 1991; 22:983-988. [PubMed: 1866765] 


\section{Table 1}

Baseline Characteristics By Case Control Status in 1990

\begin{tabular}{|c|c|c|}
\hline & Cases $(n=459)$ & Controls $(n=459)$ \\
\hline Age $^{*}$ (years) & $60.8(6.0)$ & $60.7(6.0)$ \\
\hline Median magnesium $(\mathrm{mmol} / \mathrm{l})$ & 0.86 & 0.86 \\
\hline $\operatorname{BMI}\left(\mathrm{kg} / \mathrm{m}^{2}\right)$ & $25.9(5.1)$ & $25.4(4.8)$ \\
\hline \multicolumn{3}{|l|}{ Race $^{*}$} \\
\hline - White, \% & 96.0 & 97.0 \\
\hline - African-American, $\%$ & 1.3 & 1.0 \\
\hline - Asian, \% & 1.0 & 0.7 \\
\hline - Hispanic, \% & 1.3 & 1.0 \\
\hline - Other, \% & 0.4 & 0.3 \\
\hline \multicolumn{3}{|l|}{ Smoking ${ }^{*}$} \\
\hline - Never, $\%$ & 42.0 & 42.0 \\
\hline - Past, \% & 41.0 & 42.0 \\
\hline - Current, $\%$ & 18.0 & 17.0 \\
\hline Alcohol (g/day) & $5.9(10.8)$ & $5.3(10.3)$ \\
\hline Physical Activity (METs/week) & $15.1(19.6)$ & $16.2(18.5)$ \\
\hline History of Heart Disease, $\%$ & 5.0 & 6.0 \\
\hline History of High Cholesterol, \% & 48.0 & 46.0 \\
\hline History of High Blood Pressure, \% & 48.0 & 34.0 \\
\hline History of diabetes, $\%$ & 12.0 & 6.0 \\
\hline $\mathrm{HbA} 1 \mathrm{c} \geq 6, \%$ & 17.0 & 11.0 \\
\hline \multicolumn{3}{|l|}{ Aspirin } \\
\hline - non-users, \% & 53.0 & 49.0 \\
\hline$-1-5 \mathrm{tab} / \mathrm{wk}, \%$ & 25.0 & 31.0 \\
\hline - $\geq 6 \mathrm{tab} / \mathrm{wk}, \%$ & 22.0 & 19.0 \\
\hline CRP $\geq 28.5 \mathrm{nmol} / \mathrm{l}, \%$ & 39.0 & 34.0 \\
\hline Postmenopausal Hormone Therapy use ${ }^{*} \%$ & 48.0 & 47.0 \\
\hline Thiazide diuretics, $\%$ & 24.0 & 17.0 \\
\hline
\end{tabular}

Values are means (except where indicated) \pm SD or percentages and are standardized to the age distribution of this study population

*ndicates matching factors. 


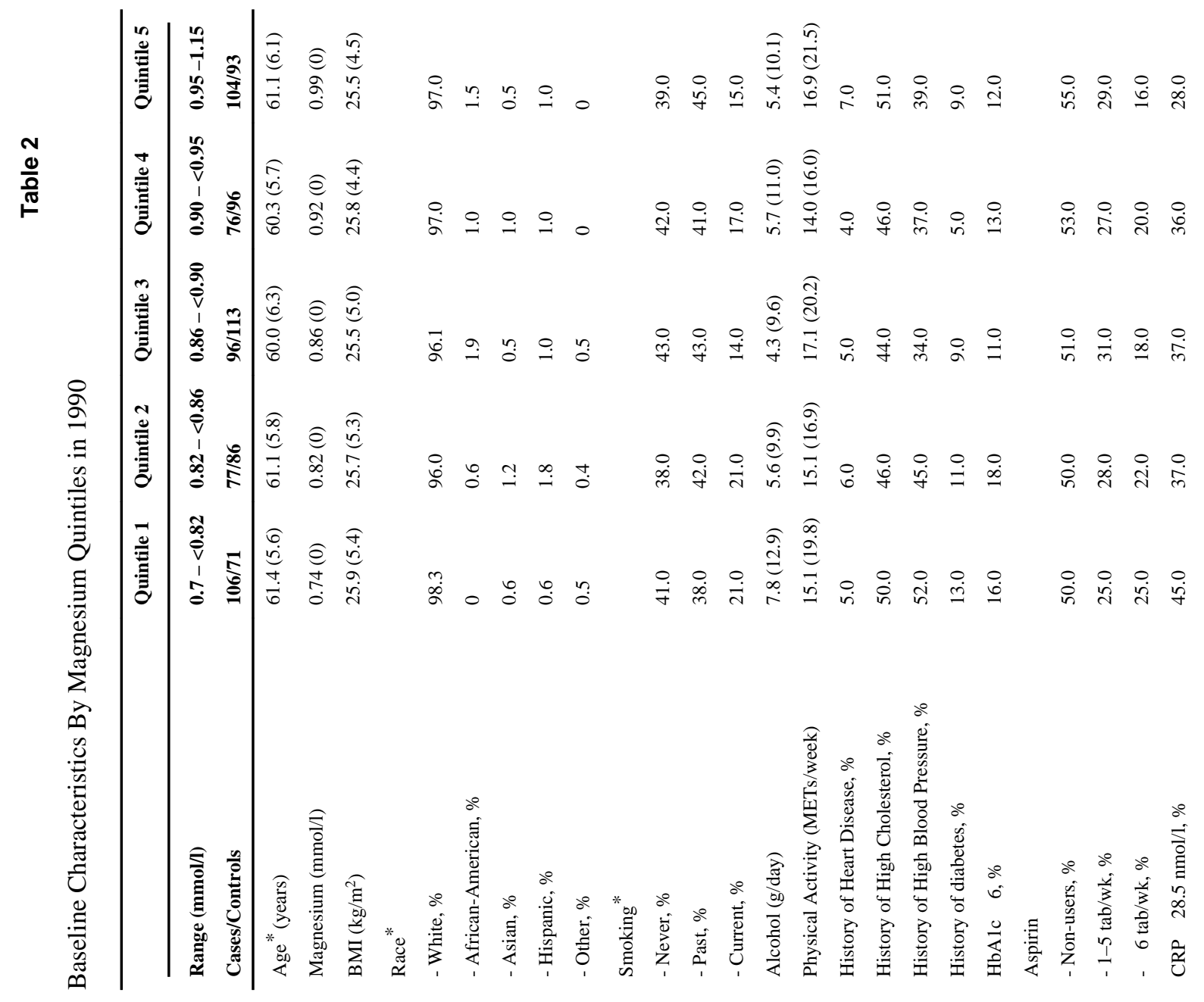




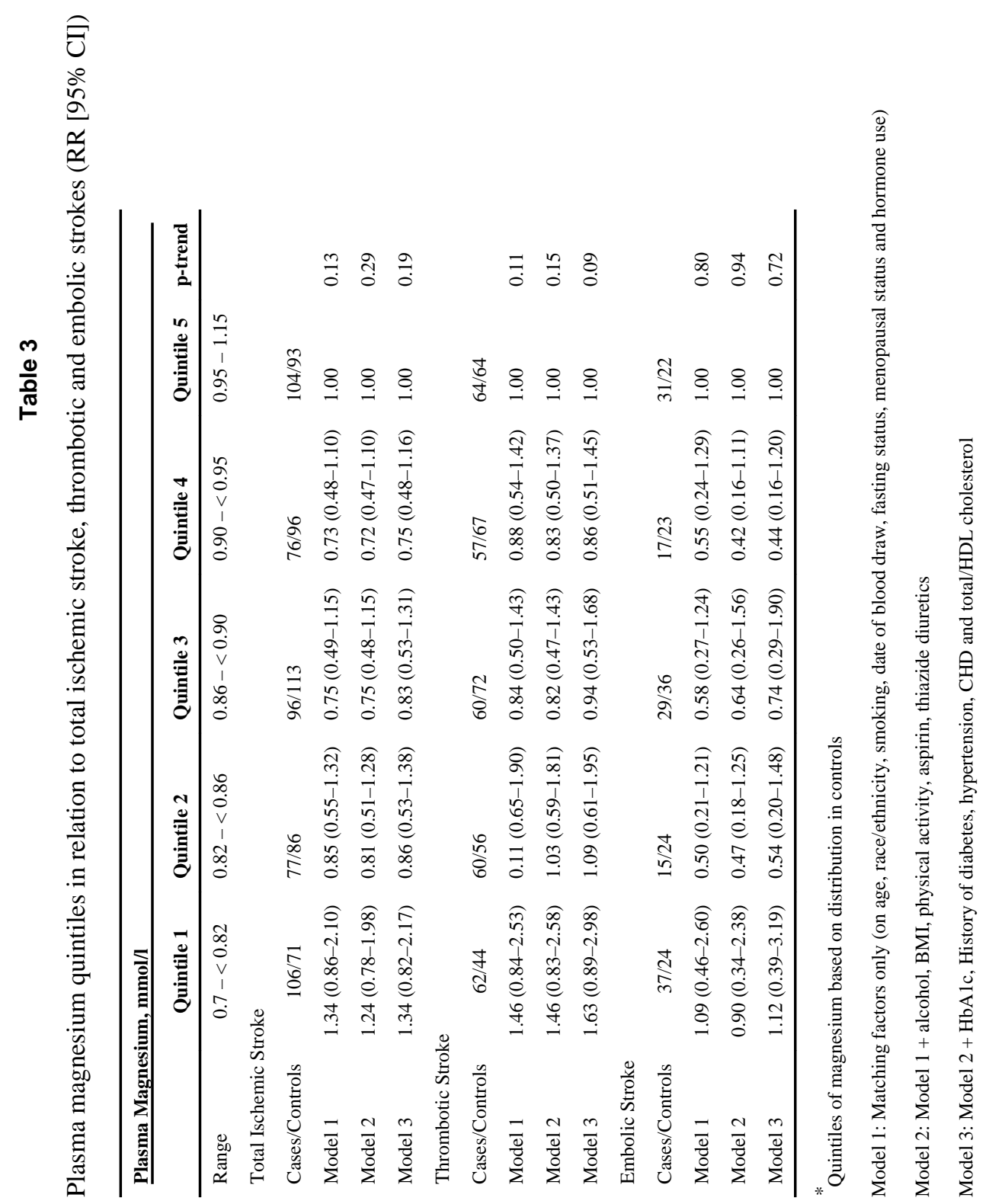


Table 4

Dichotomized magnesium levels in relation to total ischemic stroke, thrombotic and embolic strokes (RR $[95 \% \mathrm{CI}])$

\begin{tabular}{llll}
\hline & Low magnesium & High magnesium & P-value \\
\hline Magnesium levels & $<\mathbf{0 . 8 2} \mathbf{~ m m o l / l}$ & $\mathbf{2 0 . 8 2} \mathbf{~ m m o l} / \mathbf{l}$ & \\
\hline Total Stroke & & & \\
Cases/Controls & $106 / 71$ & $354 / 389$ & \\
Model 1 & $1.64(1.17-2.31)$ & 1.00 & $<0.01$ \\
Model 2 & $1.55(1.08-2.21)$ & 1.00 & 0.01 \\
Model 3 & $1.57(1.09-2.27)$ & 1.00 & 0.01 \\
Thrombotic Stroke & & & \\
Cases/Controls & $62 / 44$ & $241 / 259$ & \\
Model 1 & $1.51(0.99-2.32)$ & 1.00 & 0.05 \\
Model 2 & $1.56(1.00-2.45)$ & 1.00 & 0.05 \\
Model 3 & $1.66(1.03-2.65)$ & 1.00 & 0.03 \\
Embolic Stroke & & & \\
Cases/Controls & $37 / 24$ & $92 / 105$ & 0.05 \\
Model 1 & $1.87(0.99-3.49)$ & 1.00 & 0.22 \\
Model 2 & $1.56(0.76-3.21)$ & 1.00 & 0.14 \\
Model 3 & $1.76(0.83-3.72)$ & 1.00 &
\end{tabular}

Model 1: Matching factors only (on age, race/ethnicity, smoking, date of blood draw, fasting status, menopausal status and hormone use)

Model 2: Model $1+$ alcohol, BMI, physical activity, aspirin, thiazide diuretics

Model 3: Model $2+$ HbA1c, History of diabetes, hypertension, CHD and total/HDL cholesterol 